PROCEEDINGS OF THE

AMERICAN MATHEMATICAL SOCIETY

Volume 135, Number 1, January 2007, Pages 141-149

S 0002-9939(06)08623-0

Article electronically published on June 20, 2006

\title{
ON THE EXISTENCE OF SOLUTIONS TO THE MONGE-AMPĖRE EQUATION WITH INFINITE BOUNDARY VALUES
}

\author{
AHMED MOHAMMED
}

(Communicated by David S. Tartakoff)

\begin{abstract}
Given a positive and an increasing nonlinearity $f$ that satisfies an appropriate growth condition at infinity, we provide a condition on $g \in C^{\infty}(\Omega)$ for which the Monge-Ampère equation $\operatorname{det} D^{2} u=g f(u)$ admits a solution with infinite boundary value on a strictly convex domain $\Omega$. Sufficient conditions for the nonexistence of such solutions will also be given.
\end{abstract}

\section{INTRODUCTION}

Let $\Omega \subseteq \mathbb{R}^{n}$ be a strictly convex bounded domain and let $g$ be a positive smooth function on $\Omega$. Let $f$ be a positive and an increasing smooth function on $(\tau, \infty)$ for some $-\infty \leq \tau<\infty$. In this paper we will be concerned with convex solutions of the Monge-Ampère equation

$$
\operatorname{det} D^{2} u(x)=g(x) f(u(x)), \quad x \in \Omega,
$$

with the condition that

$$
u(x) \rightarrow \infty \text { for } x \rightarrow \partial \Omega .
$$

This problem was considered in [6, 7, 19, 20, and more recently in [14]. In all these papers, the function $g$ was assumed to be bounded on $\Omega$. Moreover, 6, 7, 19] consider the case when $f(t)=t^{\gamma}$ or when $f(t)=\exp (k t)$ for some positive $\gamma>n$ and $k$. In [14, the authors consider a much more general case with the right-hand side of (1.1) depending on the gradient $D u$ as well.

The study of solutions of elliptic equations with infinite boundary value seems to have started with the work of Bieberbach. In his 1916 paper, Bieberbach 3 considered the problem $\Delta u=\exp (u)$ in a bounded domain of the plane. Later Rademacher 24] extended his work to the case of three dimensions. It was not until 1957 that such problems were considered for general nonlinearities in arbitrary dimensions. In the papers [16, 23, Keller and Osserman studied solutions of $\Delta u=f(u)$ with infinite boundary values for general nonlinearity $f$ which satisfies a suitable growth condition at infinity. In fact they offer necessary and sufficient conditions on $f$ for such solutions to exist in bounded domains. Subsequently, related questions were posed and studied by many authors. Problem (1.1) has been studied extensively when the Monge-Ampère operator is replaced by the Laplace operator,

Received by the editors July 25, 2005.

2000 Mathematics Subject Classification. Primary 35J65, 35J60, 35J25.

(C)2006 American Mathematical Society Reverts to public domain 28 years from publication 
or more generally by a quasilinear operator. Questions of existence, uniqueness, and asymptotic boundary estimates received particular attention. We refer the reader to the papers [1, 2, 3, 8, 9, 10, 11, 13, 14, 16, 17, 18, 21, 23. and the references therein.

Given a strictly convex bounded domain $\Omega \subseteq \mathbb{R}^{n}$, we take a $g \in C^{\infty}(\Omega)$ such that $g(x)>0, \quad x \in \Omega$. We do not require $g$ to be bounded on $\Omega$. Throughout this paper we will suppose that $f \in C^{\infty}(\tau, \infty)$ for some extended real number $-\infty \leq \tau<\infty$, that $f(t) \rightarrow 0$ as $t \rightarrow \tau$, and that $f$ and $f^{\prime}$ are both positive on $(\tau, \infty)$.

Let $F$ be the antiderivative of $f$ with $F(\tau)=0$. We will need the condition

$$
\Phi(t):=\int_{t}^{\infty} \frac{1}{F(s)^{\frac{1}{n+1}}} d s<\infty
$$

for all $t>\tau$.

Whenever $f$ satisfies condition (1.3) it is known that (see [13] for a proof)

$$
\lim _{t \rightarrow \infty} \frac{F(t)^{\frac{n}{n+1}}}{f(t)}=0 .
$$

In stating sufficient conditions for the existence of solutions to problems (1.1) and (1.2), we will need the solvability of the following Dirichlet problem on $\Omega$ :

$$
\left\{\begin{aligned}
\operatorname{det}\left(D^{2} w\right) & =g & & \text { on } \Omega, \\
w & =0 & & \text { on } \partial \Omega .
\end{aligned}\right.
$$

The paper is organized as follows. In Section 2 we will state and prove some lemmas that will be used in subsequent sections. In Section 3, we state and prove an existence result to problems (1.1) and (1.2). Bounds for such solutions will also be given. In Section 4, some results on nonexistence of solutions to the problems (1.1) and (1.2) will be presented.

\section{Preliminaries}

If $f$ satisfies condition (1.3) we let $\phi$ be the inverse of the decreasing function $\Phi$ defined in (1.3). In this case we note that $\phi^{\prime}<0$ and that $\phi(s) \rightarrow \infty$ as $s \rightarrow 0+$. Direct computation shows that

$$
\left(-\phi^{\prime}\right)^{n+1}=F(\phi) \quad \text { and } \quad(n+1)(-\phi)^{n-1} \phi^{\prime \prime}=f(\phi)
$$

Furthermore, we observe

$$
\frac{-\phi^{\prime}(s)}{\phi^{\prime \prime}(s)}=(n+1) \frac{F(\phi(s))^{\frac{n}{n+1}}}{f(\phi(s))} .
$$

The following comparison lemma is well known [14, 19] and will be used repeatedly in subsequent proofs. Since we state it in a slightly different form for our purpose, we have included a short proof for completeness.

Lemma 2.1. Let $w \in C^{2}(\Omega)$ be convex, and $u \in C^{2}(\Omega)$ such that

$$
\limsup _{x \rightarrow \partial \Omega}(w(x)-u(x)) \leq 0 .
$$

If $\operatorname{det} D^{2} u<\operatorname{det} D^{2} w$ on $\Omega$, then $w \leq u$ on $\Omega$. Moreover, if $\operatorname{det} D^{2} u \leq g f(u)$ and $\operatorname{det} D^{2} w \geq g f(w)$ on $\Omega$, then $w \leq u$ on $\Omega$. 
Proof. Given $\epsilon>0$, the boundary condition (2.2) implies that $w(x) \leq u(x)+\epsilon$ for all $x \in \Omega$ with $0<\operatorname{dist}(x, \partial \Omega)<\delta$ for some $\delta>0$. We assume that the open set $G=\{x \in \Omega: w(x)>u(x)+\epsilon\}$ is nonempty, for otherwise there is nothing to show. Then $\bar{G} \subseteq \Omega$ and $w=u+\epsilon$ on $\partial G$. First let us suppose that $\operatorname{det} D^{2} u<\operatorname{det} D^{2} w$ on $\Omega$. Let the maximum of $w-u-\epsilon$ in $\bar{G}$ be attained at $x_{0} \in G$. Thus $D^{2}(w-u)\left(x_{0}\right)$ is negative semidefinite and hence $\operatorname{det} D^{2} w\left(x_{0}\right) \leq \operatorname{det} D^{2} u\left(x_{0}\right)$. But this contradicts the stated assumption, and therefore we have $w(x) \leq u(x)+\epsilon$. Let us now suppose that $\operatorname{det} D^{2} u \leq g f(u)$ and $\operatorname{det} D^{2} w \geq g f(w)$ on $\Omega$. Then since $f$ is increasing we would have $\operatorname{det} D^{2}(u+\epsilon)<g f(u+\epsilon)<g f(w) \leq \operatorname{det} D^{2} w$ on $G$ with $u+\epsilon=w$ on the boundary $\partial G$. But then the result proved earlier would imply $w \leq u+\epsilon$ on $G$, which is a contradiction. Thus, in any case, we have $w \leq u+\epsilon$ on $\Omega$, and since $\epsilon$ is arbitrary, we conclude that $w \leq u$ on $\Omega$.

We will also find the following observation useful. Let $u$ be a strictly convex $C^{2}$ function in a convex domain in $\mathbb{R}^{n}$, and let $\eta$ be a smooth function defined on an interval containing the range of $u$. If $w=\eta(u)$, then it can be shown 19, proof of Proposition 2.4] that

(2.3) $\operatorname{det} D^{2} w=\left(\eta^{\prime}(u)\right)^{n} \operatorname{det} D^{2} u+\eta^{\prime \prime}(u)\left(\eta^{\prime}(u)\right)^{n-1}\left(\operatorname{det} D^{2} u\right)(D u)^{T}\left(D^{2} u\right)^{-1} D u$, where $A^{T}$ denotes the transpose of matrix $A$.

Lemma 2.2. Let $f$ satisfy condition (1.3), and suppose $g \in C^{\infty}(\bar{\Omega})$ is positive. Then there is $h \in C^{\infty}(\Omega)$ such that $h(x) \rightarrow \infty$ as $\operatorname{dist}(x, \partial \Omega) \rightarrow 0$, and $u \leq h$ on $\Omega$ for any solution $u \in C^{\infty}(\Omega) \cap C(\bar{\Omega})$ of (1.1).

Proof. By [4, Theorem 1.1], we take $w \in C^{\infty}(\bar{\Omega})$ to be the solution of the Dirichlet problem (1.5). We put $z=-w$, and let us define $h=\phi(\epsilon z)$ for some $\epsilon>0$ to be chosen later. Then, according to (2.3), we have

$$
\begin{aligned}
\operatorname{det} D^{2} h & =\epsilon^{n}\left(\phi^{\prime}(\epsilon z)\right)^{n} \operatorname{det} D^{2} z+\epsilon^{n+1} \phi^{\prime \prime}(\epsilon z)\left(\phi^{\prime}(\epsilon z)\right)^{n-1}\left(\operatorname{det} D^{2} z\right)(D z)^{T}\left(D^{2} z\right)^{-1} D z \\
& =\epsilon^{n}\left(\operatorname{det} D^{2} w\right) \phi^{\prime \prime}(\epsilon z)\left(-\phi^{\prime}(\epsilon z)\right)^{n-1}\left[\frac{-\phi^{\prime}(\epsilon z)}{\phi^{\prime \prime}(\epsilon w)}+\epsilon(D w)^{T}\left(D^{2} w\right)^{-1} D w\right] \\
& =\frac{\epsilon^{n}}{n+1} g f(\vartheta)\left[\frac{-\phi^{\prime}(\epsilon z)}{\phi^{\prime \prime}(\epsilon z)}+\epsilon(D w)^{T}\left(D^{2} w\right)^{-1} D w\right] .
\end{aligned}
$$

Let

$$
M_{\epsilon}(x)=\frac{\epsilon^{n}}{n+1}\left[\frac{-\phi^{\prime}(\epsilon z)}{\phi^{\prime \prime}(\epsilon z)}+\epsilon(D w)^{T}\left(D^{2} w\right)^{-1} D w\right] .
$$

Taking note of the identity (2.1) and the limit (1.4), we see that, since $w \in C^{\infty}(\bar{\Omega})$, $M_{\epsilon}(x)$ can be made as small as we wish uniformly in $x \in \Omega$ by taking $\epsilon$ sufficiently small. So for such a choice of $\epsilon$, we find that

$$
\operatorname{det} D^{2} h \leq g f(h)
$$

on $\Omega$. We also note that $h(x) \rightarrow \infty$ as $\operatorname{dist}(x, \partial \Omega) \rightarrow 0$. From the comparison lemma, it follows that $u \leq h$ on $\Omega$.

\section{Existence of SOlutions to (1.1) AND (1.2)}

Theorem 3.1. Let $f$ satisfy condition (1.3) and let $g \in C^{\infty}(\Omega)$ be positive. If the Dirichlet problem (1.5) has a convex solution $w \in C^{\infty}(\Omega) \cap C(\bar{\Omega})$, then the problems (1.1) - (1.2) admit a solution $u \in C^{\infty}(\Omega)$. 
Proof. Let $w \in C^{\infty}(\Omega) \cap C(\bar{\Omega})$ be the strictly convex solution of (1.5). Since $f$ satisfies (1.3), and hence also (1.4), we define

$$
\gamma(t)=-\lambda \int_{t}^{\infty} \frac{1}{f(s)^{1 / n}} d s, \quad t>\tau
$$

so that $\gamma:(\tau, \infty) \rightarrow(\gamma(\tau), 0)$ is an increasing function. Here $\lambda \geq 1$ is chosen such that

$$
\gamma(\tau)<\min _{x \in \bar{\Omega}} w(x) .
$$

For each sufficiently big positive integer $k$, let us define the strictly convex sets

$$
\Omega_{k}:=\{x \in \Omega: w(x)<\gamma(k)\} .
$$

We now consider the Dirichlet problems:

$$
\left\{\begin{aligned}
\operatorname{det}\left(D^{2} u\right) & =\lambda^{-n} g f(u) & & \text { in } \Omega_{k}, \\
u & =k & & \text { on } \partial \Omega_{k} .
\end{aligned}\right.
$$

Any convex solution of the above problem with the right-hand side replaced by $\lambda^{-n} g f(k)$ is a subsolution of problem (3.2). Therefore, by [4, Theorem 7.1] (see also [19, Lemma 2.3]), problem (3.2) admits a strictly convex solution $u_{k}$ on $\Omega_{k}$. For $\epsilon>0$, let $v_{k}=\gamma\left(u_{k}+\epsilon\right)$ for each $k$. Note that

$$
\left(\gamma^{\prime}(t)\right)^{n}=\frac{\lambda^{n}}{f(t)} \quad \text { and } \quad \gamma^{\prime \prime}(t)\left(\gamma^{\prime}(t)\right)^{n-1}=-\frac{\lambda^{n} f^{\prime}(t)}{n f^{2}(t)} .
$$

Direct computation, where we use (3.3) in (2.3) with $w=\gamma\left(u_{k}+\epsilon\right)$, shows that

$$
\begin{aligned}
\operatorname{det} D^{2} v_{k} & =\frac{\lambda^{n} \operatorname{det} D^{2} u_{k}}{f\left(u_{k}+\epsilon\right)}-\frac{\lambda^{n} f^{\prime}\left(u_{k}+\epsilon\right) \operatorname{det} D^{2} u_{k}}{n f^{2}\left(u_{k}+\epsilon\right)}\left(D u_{k}\right)^{T}\left(D^{2} u_{k}\right)^{-1} D u_{k} \\
& =\frac{g f\left(u_{k}\right)}{f\left(u_{k}+\epsilon\right)}-\frac{f^{\prime}\left(u_{k}+\epsilon\right) \operatorname{det} D^{2} u_{k}}{n f^{2}\left(u_{k}+\epsilon\right)}\left(D u_{k}\right)^{T}\left(D^{2} u_{k}\right)^{-1} D u_{k} .
\end{aligned}
$$

Since $u_{k}$ is strictly convex and $\operatorname{det} D^{2} u_{k}>0$, we see that $D^{2} u_{k}$ and hence its inverse $\left(D^{2} u_{k}\right)^{-1}$ is positive definite. This together with the fact that $f$ is increasing leads to

$$
\operatorname{det} D^{2} v_{k}<g
$$

on $\Omega_{k}$. As $w$ is a solution of (1.5) on $\Omega$, we conclude that

$$
\operatorname{det} D^{2} v_{k}<\operatorname{det} D^{2} w
$$

on $\Omega_{k}$. Since $w \leq v_{k}=\gamma\left(u_{k}+\epsilon\right)$ on $\partial \Omega_{k}$, it follows from the comparison lemma that $w \leq \gamma\left(u_{k}+\epsilon\right)$ on $\Omega_{k}$. Therefore, since $\epsilon$ is arbitrary, we have $\gamma^{-1}(w) \leq u_{k}$ on $\Omega_{k}$. Because $u=\gamma^{-1}(w)$ on $\Omega_{k}$ and $\gamma^{-1}(w) \leq u_{k+1}$ on $\Omega_{k+1}$, it follows from the comparison lemma that $u_{k} \leq u_{k+1}$ on $\Omega_{k}$.

Now, let $x_{0} \in \Omega$, and let $N$ be a positive integer so that $x_{0} \in \Omega_{N}$. Since $g \in C^{\infty}\left(\bar{\Omega}_{N+1}\right)$, by Lemma 2.2, we pick $h_{N} \in C^{\infty}\left(\Omega_{N+1}\right)$ such that $u_{k} \leq h_{N}$ for all $k \geq N$. Thus $\left\{u_{k}\left(x_{0}\right)\right\}$, being an increasing sequence that is bounded by $h\left(x_{0}\right)$, converges to a limit $u\left(x_{0}\right)$. We now proceed to show that the limit function $u$ so obtained is a solution of (1.1). The argument rests on well-known a priori estimates for solutions of (3.2) established in the papers [4, 26]. Such arguments have been used in [19] when $\tau>-\infty$ and in [14, 20, 25] for the case $\tau=-\infty$. For completeness we will provide the argument when $\tau>-\infty$ in our case. In fact it is no loss of generality to suppose that $\tau=0$ in this situation, for otherwise we consider problems (1.1) - (1.2) with $f(t)$ replaced by $f(t+\tau)$. 
Let $m=\min \left\{\gamma^{-1}(w)(x): x \in \bar{\Omega}_{N}\right\}$ and $M=\max \left\{h_{N}(x): x \in \bar{\Omega}_{N}\right\}$. Then $m \leq u_{k} \leq M$ on $\Omega_{N}$ for all $k>N$. By [26, Proposition 2.4(ii)] (or [19, Lemma 2.2]), there is a constant $C$ that depends only on $m, M$, bounds of $g$ and its derivatives on $\Omega_{N}$, bounds of $f$ and its derivatives on $[m, M]$, and $\operatorname{dist}\left(\Omega_{N}, \partial \Omega_{k}\right)$ such that

$$
\left\|u_{k}\right\|_{C^{3}\left(\bar{\Omega}_{N}\right)} \leq C
$$

for $k>N$. Since for such $k$,

$$
0<\operatorname{dist}\left(\Omega_{N}, \partial \Omega_{N+1}\right) \leq \operatorname{dist}\left(\Omega_{N}, \partial \Omega_{k}\right) \leq \operatorname{dist}\left(\Omega_{N}, \partial \Omega\right),
$$

the constant $C$ can actually be chosen to be independent of $k$. Hence, the sequence $\left\{u_{k}\right\}$ contains a subsequence that converges uniformly, together with its first and second derivatives, to $u$ and therefore $\operatorname{det}\left(D^{2} u\right)=g f(u)$. From elliptic regularity theory it follows that $u \in C^{\infty}(\Omega)$. Since $\gamma^{-1}(w) \leq u$ on $\Omega$ and $\gamma^{-1}(0-)=\infty$, we see that $u$ has infinite value on the boundary $\partial \Omega$.

Corollary 3.2. Let $f$ satisfy (1.3) and let $g$ be a smooth function on $\Omega$ such that $0<g(x) \leq A \operatorname{dist}(x, \partial \Omega)^{\delta-n-1}$ for some positive constants $\delta$ and $A$. Then the problems (1.1)-(1.2) admit a smooth solution on $\Omega$.

Proof. It is shown in [5. Theorem 3] that under the given conditions on $g$, the Dirichlet problem (1.5) has a unique convex solution $w \in C^{\infty}(\Omega) \cap C^{\epsilon}(\bar{\Omega})$ for some $\epsilon>0$. Thus the corollary follows from the above theorem.

Remark 3.3. The existence of a solution to (1.1)-(1.2), when $f$ satisfies (1.3) and $g \in C^{\infty}(\bar{\Omega})$ is positive, has already been established in the papers 20 , 25. See also [18 for an existence result when $f(t)=t^{\gamma}, \gamma>n$, or $f(t)=e^{t}$ and $g \in C^{\infty}(\bar{\Omega})$. Thus Theorem 3.1 generalizes these results to the case when $g$ is not necessarily bounded on $\Omega$.

Theorem 3.4. Let $f$ satisfy condition (1.3) and let $g \in C^{\infty}(\Omega)$ be a positive function such that the Dirichlet problem (1.5) has a convex solution. If, in addition, $\inf \{g(x): x \in \Omega\}>0$ and $g \in L^{1}(\Omega)$, then there are functions $h_{1}, h_{2}:(0, \infty) \rightarrow \mathbb{R}$ with $h_{j}(r) \rightarrow \infty$ as $r \rightarrow 0$ such that for any solution $u$ of problems (1.1)-(1.2),

$$
h_{1}(d(x, \partial \Omega)) \leq u(x) \leq h_{2}(d(x, \partial \Omega)), \quad x \in \Omega .
$$

Proof. Let $w \in C^{\infty}(\Omega) \cap C(\bar{\Omega})$ be a solution of (1.5). Then, by Alexandrov's maximum principle [15, Theorem 1.4.2], we have

$$
w(x) \geq-C d(x, \partial \Omega)^{1 / n}, \quad x \in \Omega,
$$

where $C$ is a positive constant that depends on the diameter $\operatorname{diam}(\Omega)$ of $\Omega$, the dimension $n$, and the $L^{1}$ norm of $g$ on $\Omega$. In the definition of $\gamma$ given in (3.1), we choose $\lambda \geq 1$ such that $\gamma(\tau+)<-C(\operatorname{diam}(\Omega))^{1 / n}$. Now if $u$ is any solution of (1.1) - (1.2) on $\Omega$, then arguing as in the proof of Theorem 3.1, one can show that $w \leq \gamma(u+\epsilon)$ on $\Omega$ for any $\epsilon>0$. Thus,

$$
\gamma^{-1}\left(-C d(x, \partial \Omega)^{1 / n}\right) \leq \gamma^{-1}(w) \leq u+\epsilon, \quad x \in \Omega .
$$

Since $\epsilon$ is arbitrary, the left-hand side inequality in (3.4) follows with $h_{1}(t)=$ $\gamma^{-1}\left(-C t^{1 / n}\right)$. We now proceed to establish the right-hand side inequality. 
Given $x_{0} \in \Omega$, let $z(x)=\left(r^{2}-\left|x-x_{0}\right|^{2}\right) / 2$ for $0<r \leq \operatorname{diam}(\Omega)$. An application of (2.3) to the function $\vartheta(x)=\phi(\epsilon z(x))$, where $\epsilon>0$ is to be determined, shows that

$$
\begin{aligned}
\operatorname{det} D^{2} \vartheta & =\epsilon^{n}\left(\phi^{\prime}(\epsilon z)\right)^{n} \operatorname{det} D^{2} z+\epsilon^{n+1} \phi^{\prime \prime}(\epsilon z)\left(\phi^{\prime}(\epsilon z)\right)^{n-1}\left(\operatorname{det} D^{2} z\right)(D z)^{T}\left(D^{2} z\right)^{-1} D z \\
& =\epsilon^{n} \phi^{\prime \prime}(\epsilon z)\left(-\phi^{\prime}(\epsilon z)\right)^{n-1}\left[\frac{-\phi^{\prime}(\epsilon z)}{\phi^{\prime \prime}(\epsilon z)}+\epsilon\left|x-x_{0}\right|^{2}\right] \\
& =\frac{\epsilon^{n}}{n+1} f(\vartheta)\left[\frac{-\phi^{\prime}(\epsilon z)}{\phi^{\prime \prime}(\epsilon z)}+\epsilon\left|x-x_{0}\right|^{2}\right] .
\end{aligned}
$$

Let

$$
M_{\epsilon}(x ; r):=\frac{\epsilon^{n}}{n+1}\left[\frac{-\phi^{\prime}(\epsilon z)}{\phi^{\prime \prime}(\epsilon z)}+\epsilon\left|x-x_{0}\right|^{2}\right] .
$$

Let $m_{g}>0$ be the infimum of $g$ on $\Omega$. As in the proof of Lemma 2.2, we take $\epsilon$ sufficiently small so that $M_{\epsilon}(x, r) \leq m_{g}$ for $x \in \Omega$ and $0 \leq r \leq \operatorname{diam}(\Omega)$. Thus

$$
\operatorname{det} D^{2} \vartheta-g f(\vartheta) \leq\left[M_{\epsilon}(x, r)-m_{g}\right] f(\vartheta)<0, \quad x \in B\left(x_{0}, r\right) .
$$

Since $\vartheta=\infty$ on $\partial B\left(x_{0}, r\right)$, by the comparison lemma it follows that $u \leq \phi(\epsilon z)$ on $B\left(x_{0}, r\right) \subseteq \Omega$ for $0<r \leq d\left(x_{0}, \partial \Omega\right)$. In particular, $u\left(x_{0}\right) \leq \phi\left(\epsilon r^{2} / 2\right)$. Thus given $x_{0} \in \Omega$, we have $u\left(x_{0}\right) \leq h_{2}\left(d\left(x_{0}, \partial \Omega\right)\right)$, where $h_{2}(r)=\phi\left(\epsilon r^{2} / 2\right)$ and $r$ is taken to be $d\left(x_{0}, \partial \Omega\right)$.

\section{Nonexistence of SOlutions to (1.1) And (1.2)}

For our first nonexistence result, we consider a class of positive functions $g \in$ $C^{\infty}(\Omega)$ such that

$$
\liminf _{x \rightarrow x_{0}} g(x)\left|x-x_{0}\right|^{2 n}=\alpha
$$

for some $x_{0} \in \partial \Omega$ and some $\alpha \in(0, \infty]$.

Theorem 4.1. Suppose $f$ satisfies (1.3) and that $g \in C^{\infty}(\Omega)$ is a positive function that satisfies (4.1) for some $x_{0} \in \partial \Omega$ and some $0<\alpha \leq \infty$. Then there is no convex function $u \in C^{2}(\Omega)$ that solves the problems (1.1) and (1.2) on $\Omega$.

Proof. By hypothesis, there are positive numbers $\beta$ and $\delta$ such that

$$
g(x) \geq \beta\left|x-x_{0}\right|^{-2 n}
$$

for $x$ in $B\left(x_{0}, \delta\right) \cap \Omega$. Here, $B\left(x_{0}, \delta\right)$ stands for the ball of radius $\delta$ centered at $x_{0}$. Let $x_{0}^{r}=x_{0}-2 r \vec{n}\left(x_{0}\right)$, where $\vec{n}\left(x_{0}\right)$ is the outer unit normal vector to $\partial \Omega$ at $x_{0}$. For $0<r<\delta / 3$, we note that $\overline{B\left(x_{0}^{r}, r\right)} \subseteq B\left(x_{0}, \delta\right) \cap \Omega$, and therefore, $g(x) \geq \beta r^{-2 n}$ for $x \in B\left(x_{0}^{r}, r\right)$. We now let $\vartheta(x)=\phi(\epsilon z(x))$, where

$$
z(x)=\frac{1}{2}\left(1-\frac{\left|x-x_{0}^{r}\right|^{2}}{r^{2}}\right), \quad x \in B\left(x_{0}^{r}, r\right),
$$

and $\epsilon>0$ is to be determined.

Computing as in the proof of Lemma 2.2 we find that for $x \in B\left(x_{0}^{r}, r\right)$,

$$
\begin{aligned}
\operatorname{det} D^{2} \vartheta & =\frac{\epsilon^{n}}{(n+1) r^{2 n}} f(\vartheta)\left[\frac{-\phi^{\prime}(\epsilon z)}{\phi^{\prime \prime}(\epsilon z)}+\epsilon \frac{\left|x-x_{0}^{r}\right|^{2}}{r^{2}}\right] \\
& \leq \frac{\epsilon^{n}}{(n+1) r^{2 n}} f(\vartheta)\left[\frac{-\phi^{\prime}(\epsilon z)}{\phi^{\prime \prime}(\epsilon z)}+\epsilon\right] .
\end{aligned}
$$


Thus, for sufficiently small $\epsilon$, we conclude as in the proof of Lemma 2.2 that

$$
\operatorname{det} D^{2} \vartheta \leq \frac{\epsilon^{n}}{r^{2 n}} f(\vartheta), \quad x \in B\left(x_{0}^{r}, r\right) .
$$

Therefore, we observe that

$$
\operatorname{det} D^{2} \vartheta-g f(\vartheta) \leq \frac{1}{r^{2 n}} f(\vartheta)\left[\epsilon^{n}-\beta\right],
$$

on the ball $B\left(x_{0}^{r}, r\right)$. Thus, we see that $\operatorname{det} D^{2} \vartheta<g f(\vartheta)$ on $B\left(x_{0}^{r}, r\right)$ for $\epsilon$ small enough. Consequently, since $u$ satisfies (1.1) on $\Omega$, we have $\operatorname{det} D^{2} u>\operatorname{det} D^{2} \vartheta$ on $B\left(x_{0}^{r}, r\right)$. Note that $\vartheta(x)=\infty$ on $\partial B\left(x_{0}^{r}, r\right)$. Since $u \in C^{2}(\Omega)$ and $\overline{B\left(x_{0}^{r}, r\right)} \subseteq \Omega$, for sufficiently small $r$, by the comparison lemma we have $u \leq \vartheta$ on $B\left(x_{0}^{r}, r\right)$. In particular we have

$$
u\left(x_{0}^{r}\right) \leq \phi(\epsilon / 2) .
$$

Thus $u\left(x_{0}-2 r \vec{n}\left(x_{0}\right)\right) \leq \phi(\epsilon)$, and therefore $\limsup _{r \rightarrow 0^{+}} u\left(x_{0}-2 r \vec{n}\left(x_{0}\right)\right) \leq \phi(\epsilon / 2)$.

In the paper 19, the authors show that problems (1.1) and (1.2) have no solution when $g \in C^{\infty}(\bar{\Omega})$ and $f(t)=t^{\gamma}, 0<\gamma \leq n$. The next result extends this nonexistence result to include $g \in C^{\infty}(\Omega)$ and nonlinearities $f$ that satisfy the following condition:

$$
\int_{1}^{\infty} \frac{1}{f(t)^{1 / n}} d t=\infty
$$

Theorem 4.2. Suppose $f$ satisfies (4.2). If $\operatorname{det} D^{2} w \geq g$ on $\Omega$ for some convex function $w \in C^{2}(\Omega) \cap C(\bar{\Omega})$, then there is no strictly convex function $u \in C^{2}(\Omega)$ such that $\operatorname{det} D^{2} u \leq g f(u)$ on $\Omega$ and that satisfies (1.2).

Proof. Suppose $u \in C^{2}(\Omega)$ is a strictly convex function that satisfies $\operatorname{det} D^{2} u \leq$ $g f(u)$ on $\Omega$ and the boundary condition (1.2). We choose $\alpha>0$ such that $u+\alpha \geq 1$ on $\Omega$. Let

$$
\mu(t)=\int_{1}^{t} \frac{1}{f(s)^{1 / n}} d s, \quad t>1
$$

By hypothesis, we recall that $\mu(\infty)=\infty$. Let $v(x)=\mu(u(x)+\alpha), \quad x \in \Omega$.

Then as before, we see that

$$
\operatorname{det} D^{2} v=\frac{\operatorname{det} D^{2} u}{f(u+\alpha)}-\frac{1}{n} \cdot \frac{f^{\prime}(u+\alpha)}{f^{2}(u+\alpha)}\left(\operatorname{det} D^{2} u\right)(D u)^{T}\left(D^{2} u\right)^{-1} D u .
$$

Since $u$ is convex and $f$ is increasing, it follows that $\operatorname{det} D^{2} v<g$ on $\Omega$. As a consequence, we have $\operatorname{det} D^{2} v<\operatorname{det} D^{2} w$ on $\Omega$. Now let $\beta \geq 0$ be an arbitrary real number. Then $\operatorname{det} D^{2} v<\operatorname{det} D^{2}(w+\beta)$. Since $w+\beta \leq v$ on $\partial \Omega$, from the comparison principle we conclude that $w+\beta \leq v$ on $\Omega$. Thus it has been shown that

$$
v-w \geq \beta
$$

on $\Omega$ for any real number $\beta$, which is an obvious contradiction. 


\section{REFERENCES}

[1] C. Bandle, M. Marcus, Large solutions of semilinear elliptic equations: Existence uniqueness, and asymptotic behavior, J. Anal. Math., 58 (1992), 9-24. MR1226934 (94c:35081)

[2] C. Bandle, M. Marcus, Asymptotic behavior of solutions and their derivatives for semilinear elliptic problems with blowup on the boundary, Ann. Inst. Henri Poincaré, 12 (1995), 155-171. MR.1326666 (96e:35038)

[3] L. Bieberbach, $\Delta u=e^{u}$ und die authomorphen funktionen, Math. Ann., 77 (1916), 173-212. MR 1511854

[4] L. Caffarelli, L. Nirenberg, and J. Spruck, The Dirichlet problem for nonlinear second order elliptic eqautions I. Monge-Ampère equations, Comm. Pure Appl. Math., 37 (1984), 369-402. MR.0739925 (87f:35096)

[5] S. Y. Cheng and S.-T. Yau, On the regularity of the Monge-Ampère equation $\operatorname{det}\left(\left(\partial^{2} u / \partial x^{i} \partial x^{j}\right)\right)=F(x, u)$, Comm. Pure Appl. Math., 30 (1997), 41-68. MR0437805 $(55: 10727)$

[6] S. Y. Cheng and S.-T. Yau, On the existence of a complete Kähler metric on noncompact complete manifolds with the regularity of Fefferman's equation, Comm. Pure Appl. Math., 33 (1980), 507-544. MR0575736 (82f:53074)

[7] S. Y. Cheng and S.-T. Yau, The real Monge-Ampère equation and affine flat structure, Proc. 1980 Beijing Symp. on Diff. Geom. and Diff. Equations, Vol. I (S. S. Chern and W. T. Wu, eds.), Science Press, Beijing (1982), 339-370. MR0714338 (85c:53103)

[8] F.-C. Cîrstea, V. Rădulescu, Existence and uniqueness of blow-up solutions for a class of logistic equations, Commun. Contemp. Math., 4 (2002), 559-586. MR1918760 (2003f:35120)

[9] G. Diaz, R. Letelier, Explosive solutions of quasilinear elliptic eqiuations: Existence and Uniqueness, Nonlinear Analysis, 20 (1993), 97-125. MR1200384 (94a:35017)

[10] U. Du, Q. Huang, Blow-up solutions and their applications in quasilinear elliptic equations, J. Anal. Math., 89 (2003), 277-302. MR1981921 (2004c:35123)

[11] J. Garcia-Melián, R. Letelier, J. S. de Lis, Uniqueness and asymptotic behaviour for functions of semilinear problems with boundary blow-up, Proc. Amer. Math. Soc., 129 (2001), 35933602. MR.1860492 (2002j:35117)

[12] D. Gilbarg and N. Trudinger, Elliptic Partial Differential Equations of Second Order, 2nd ed., Revised 3rd printing, Springer-Verlag, 1998. MR1814364 (2001k:35004)

[13] F. Gladiali, G. Porru, Estimates for explosive solutions to p-Laplace equations, Progress in Partial Differential Equations (Pont-á-Mousson 1997), Vol. 1, Pitman Res. Notes Math. Series, Longman 383 (1998), 117-127. MR.1628068 (2000h:35047)

[14] B. Guan and H.-Y. Jian, The Monge-Ampère equation with infinite boundary value, Pacific J. Math., 216 (2004), 77-94. MR2094582 (2005f:35100)

[15] C. E. Gutierrez, The Monge-Ampère Equation, Birkhaüser, Berlin, 2001. MR1829162 (2002e:35075)

[16] J. B. Keller, On solutions of $\Delta u=f(u)$, Comm. Pure Appl. Math., 10 (1957), 503-510. MR0091407 (19:964c)

[17] A. V. Lair, A necessary and sufficient condition for existence of large solutions to semilinear elliptic equations, J. Math. Anal. Appl., 240 (1999), 205-218. MR.1728197(2000i:35046)

[18] A. C. Lazer, P. J. McKenna, Asymptotic behaviour of solutions of boundary blow up problems, Differential and Integral Equations, 7 (1994), 1001-1019. MR1270115 (95c:35084)

[19] A. C. Lazer, P. J. McKenna, On singular boundary value problems for the Monge-Ampère operator, J. Math. Anal. Appl., 197 (1996), 341-362. MR1372183 (97c:35064)

[20] J. Matero, The Bieberbach-Rademacher problem for the Monge-Ampère operator, Manuscripta Math., 91 (1996), 379-391. MR.1416719 (97i:35107)

[21] J. Matero, Quasilinear elliptic equations with boundary blow-up, J. Anal. Math., 69 (1996), 229-247. MR:1428101 (97m:35089)

[22] A. Mohammed, Existence and asymptotic behavior of blow-up solutions to weighted quasilinear equations, J. Math. Anal. Appl., 298 (2004), 621-637. MR2086979 (2005f:35109)

[23] R. Osserman, On the inequality $\Delta u \geq f(u)$, Pacific J. Math., 7 (1957), 1641-1647. MR0098239 (20:4701)

[24] H. Rademacher, Einige besondere probleme partieller Differentialgleichun (1943), 838-845. 
[25] P. Salani, Boundary Blow-up problems for Hessian equations, Manuscripta Math., 96 (1998), 281-294. MR.1638149 (99e:35071)

[26] K. Tso, On a real Monge-Ampère functional, Invent. Math., 101 (1990), 425-448. MR.1062970 (91i:35082)

Department of Mathematical Sciences, Ball State University, Muncie, Indiana 47306

E-mail address: amohammed@bsu.edu 